\title{
Brief cognitive-behavioural therapy for patients in the community with schizophrenia: randomised controlled trial in Beijing, China
}

\author{
Zhi-Hua Guo, Zhan-Jiang Li, Yun Ma, Jing Sun, Jun-Hua Guo, Wen-Xiu Li, Zhi-Qiang Wang, \\ Hui-Li Xu, Roger M. K. Ng, Douglas Turkington and David Kingdon
}

\section{Background}

Brief cognitive-behavioural therapy (CBT) is an emerging treatment for schizophrenia in community settings; however further trials are needed, especially in non-Western countries.

\section{Aims}

To test the effects of brief CBT for Chinese patients with schizophrenia in the community (trial registration: ChicTRTRC-13003709).

\section{Method}

A total of 220 patients with schizophrenia from four districts of Beijing were randomly assigned to either brief CBT plus treatment as usual (TAU) or TAU alone. Patients were assessed at baseline, post-treatment and at 6- and 12-month follow-ups by raters masked to group allocation.

\section{Results}

At the post-treatment assessment and the 12 -month folle up, patients who received brief CBT showed greater improvement in overall symptoms, general psychopathology, insight and social functioning. In total, 37.3\% of those in the brief CBT plus TAU group experienced a clinically significant response, compared with only $19.1 \%$ of those in the $\mathrm{MAU}$ alone group $(P=0.003)$

\section{Conclusions}

Brief CBT has

schizoph

\section{Declaration of interest}

\section{None.}

\section{Copyright and usage}

(c) The Royal college of Psychiatrists 2017.
Schizophrenia is a severe and disabling mental disorder. However, the potential for recovery is increasingly being recognised. ${ }^{1} \mathrm{Ap}$ epidemiological study in 1998 reported a $0.65 \%{ }^{2}$ and it is estimated that there are people with schizophrenia in mainland lifelong antipsychotic medication people still have residual sym insight and adherence to tre rate. ${ }^{4}$ Schizophrenia functioning in their da increasing interest in community-based rehabilitation in China. Psychological intervention has been an important part of community mental healthcare. Cognitive-behavioural therapy (CBT) has become one of the most extensively studied psychological interventions in the treatment of schizophrenia in Western countries. 6 Both the National Institute for Health and Care Excellence (NICE) and the schizophrenia Patient Outcome Research Team $\left(\right.$ PORT) ${ }^{8}$ have recommended CBT as a routine treatment for schizophrenia to supplement the limitations of medication. A multicentre randomised controlled trial (RCT) conducted in psychatric hospitals in China has also demonstrated that CBT can effectively reduce the symptoms of patients with schizophrenia and enhance their insight and social functioning. ${ }^{9}$ CBT for schizophrenia is a complex intervention often delivered by highly trained and experienced therapists. However, people with schizophrenia in China still have poor access to CBT because of a lack of such staff.

There is an increasing need to integrate CBT into daily care because in China over $90 \%$ of patients are living with their family in the community and contact between patients and community staff is more frequent than those with the tertiary hospital psychiatrists. ${ }^{10}$ In Western countries, low-intensity CBT manuals suitable for community staff have been demonstrated to have good effects on relieving patients' depression and distress, improving their insight and negative symptoms. ${ }^{11-13}$ However, no brief CBT programme specifically for Chinese patients with schizophrenia in the community has been developed and whether the benefits of such a programme can be replicated in China has not yet been tested. The present study, therefore, aimed to develop a community-based brief CBT manual for Chinese patients with schizophrenia and to test the hypothesis that brief CBT has a beneficial effect on psychopathology, insight, mood and social functioning in Chinese patients in the community.

\section{Method}

\section{Study design and participants}

This single-blind RCT was conducted between 8 May 2011 and 30 October 2013 at six community care centres in four districts (Xicheng, Dongcheng, Chaoyang and Haidian) of Beijing. Outpatients were consecutively referred to the study by community mental health teams if they: (a) had a diagnosis of schizophrenia according to ICD-10 criteria; ${ }^{14}$ (b) were aged between 18 and 60 years; (c) were receiving treatment with a single antipsychotic medication at a stable dose for more than 4 weeks; and (d) had the ability to communicate and sign a consent form. Individuals were excluded if they: (a) were agitated and needing in-patient care or intensive home treatment (they might be deteriorating and there would be a change in medication); (b) had a primary diagnosis of intellectual disability or drug/alcohol dependence; (c) scored at least 5 on the Positive and Negative Syndrome Scale (PANSS $)^{15}$ in conceptual disorganisation, poor rapport or lack of spontaneity and flow of conversation (it was difficult to communicate with them); (d) had received electroconvulsive therapy (ECT) in the 6 months prior to entry into the study 
(ECT could have an impact on their memory function); or (e) were currently receiving other types of systematic psychotherapy. Participation was discontinued at any point during the study if the person was judged to be at a high risk for suicide. They were also discontinued if they were admitted to hospital or experiencing severe adverse events. The study protocol and consent form were approved by the Human Research and Ethics Committee of Beijing Anding Hospital affiliated to Capital Medical University (Year 2011, No. 3). All participants and their guardians gave written informed consent. The trial was registered with the China Clinical Trial Centre: ChiCTR-TRC-13003709. The trial flow chart is illustrated in Fig. 1.

\section{Sample size and randomisation}

In a previous preliminary trial, the effective rates of brief CBT plus treatment as usual (TAU) and TAU alone were $59.4 \%$ and $28.1 \%$, respectively $^{16}$ ('effectiveness' was defined as having a $25 \%$ or greater reduction in PANSS total score). Based on this group difference, it was calculated that at least 88 participants were required in each group to achieve a significance level of $5 \%$ (two sided) and a power of $80 \%$. Assuming a $20 \%$ drop-out rate, we planned to recruit 220 participants into this study.

Participants were randomly assigned (ratio 1:1) to the brief CBT plus TAU group or the TAU alone group. Randomisation was conducted by computer-generated (SPSS Version 20.0) blocks of four random numbers and stratified by site. Randomisation was performed by an independent researcher who was not involved in either outcome assessment or patient treatment. To preserve the masking of the ratings, all participants were instructed by the research coordinator not to disclose their treatment allocation to the raters at any time and some randomly selected patient from the TAU group were sent a sample of CBT material.

\section{Intervention}

Brief CBT

All of the patients were treated brief CBT intervention was comprising eight sessions lasting $60 \mathrm{~min}$. The $\mathrm{ma}$ Kingdon $^{11}$ and tailored to Chinese patients with schizophrenia in the community. was piloted with 16 patients to test that it had good acceptability to both the patients and therapists.

The main goals of the brief CBT included helping patients to learn coping skills, reducing their distress, increasing their activities and preyenting relapse. By focusing on stress and coping behaviours, the intervention can be disseminated into primary care more easily. The first three sessions were delivered within 2 weeks and the remaining five sessions were offered once every 2 weeks, which could be divided into three stages. In the first stage (sessions 1-2) therapists listened carefully to patients and built a good rapport. Assessment of patients' life experience, development of patients' problem list and normalisation of psychotic symptoms were also undertaken in the initial sessions. The intermediate stage (sessions 3-6) involved therapists helping the patients to learn cognitive and behavioural coping skills. For residual delusions, behavioural experiments and graded exposure were used to reduce fear and avoidance. Interventions to reduce stress from residual auditory hallucinations included reattribution of voices and coping strategies. Activity schedules and graded task assignments were applied to improve negative symptoms. In the last stage (sessions 7-8), therapists focused on problem-solving skills, discussing the advantages and disadvantages of medication,

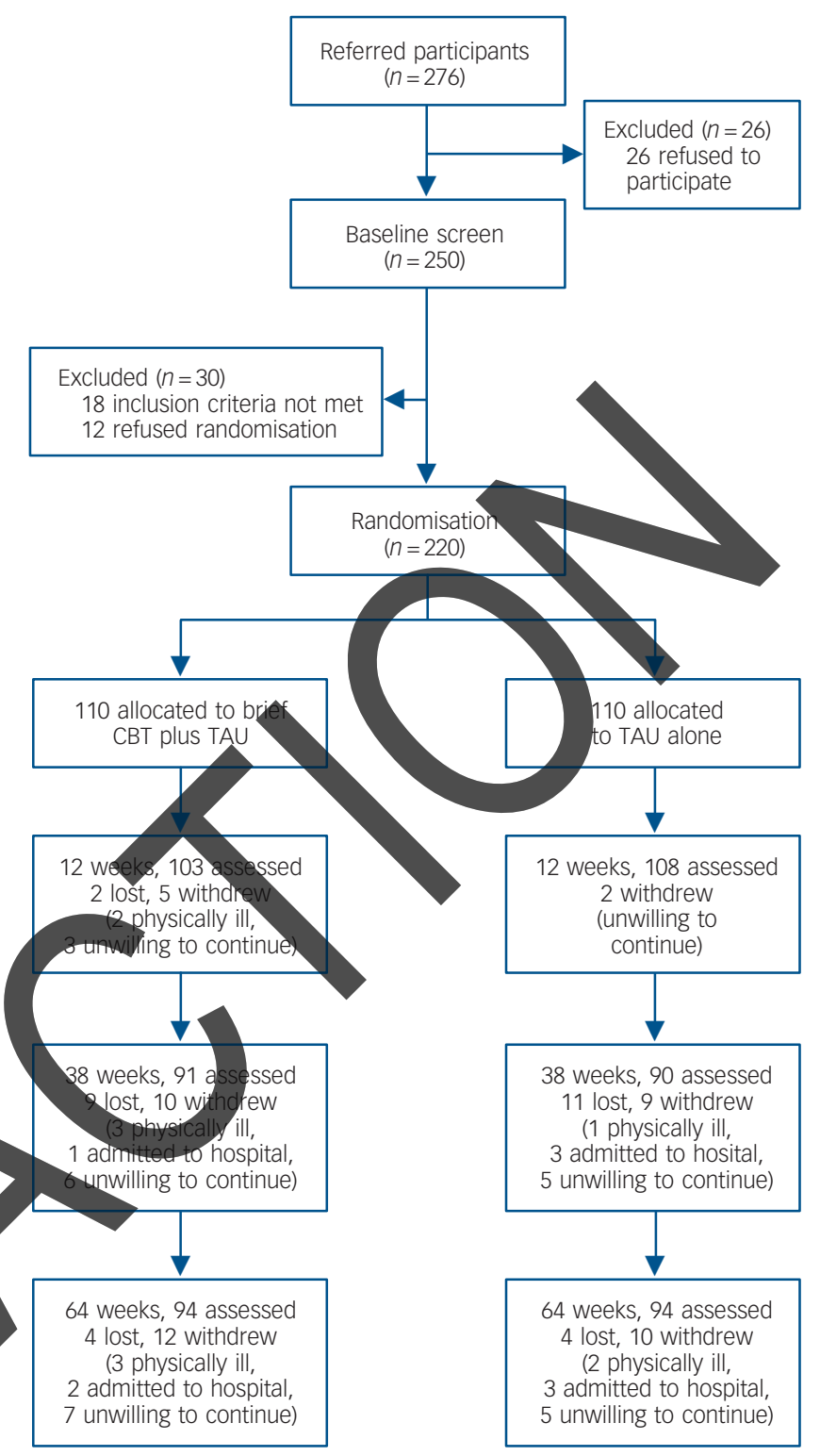

Fig. 1 CONSORT (Consolidated Standards of Reporting Trials) diagram.

CBT, cognitive-behavioural therapy; TAU, treatment as usual; 'lost' refers to moving away or being out of contact.

and learning to identify and cope with recurrence if any warning signs emerged.

Homework was assigned in a flexible and relaxed manner after each session to help participants consolidate what they had learned in the treatment. The homework included reviewing therapy notes, completing daily activity schedules, undertaking behavioural experiments and reading self-help brochures. Therapists had to check patients' homework and discuss it at the beginning of each session to ensure that patients had mastered the skills learned in previous sessions.

\section{TAU}

Participants in both arms received TAU from community clinicians. The goal of TAU is to maximise drug efficacy and improve patients' social skills. TAU included the spectrum of all the available services, similar in the four districts, not only 
antipsychotic pharmacotherapy but also clinical case management, psychological health education, social support and rehabilitation activities. Community psychiatrists and nurses visited patients at least once every month to evaluate patients, establish a safe and effective medical treatment plan, encourage patients' medication adherence and help family members to detect adverse drug reactions. ${ }^{17}$ At the same time, patients were encouraged to participate in the rehabilitation activities at local community centres. When patients became seriously unwell or there were serious adverse drug reactions an emergency response was immediately provided. However, case management in China is not as good as that in some Western countries, because of the lack of strict division and cooperation among community clinicians, psychologists, social workers, nurses and police.

\section{Therapists and fidelity}

A total of 12 trained community clinicians or nurses served as the therapists responsible for delivering the brief CBT programme. Each therapist had at least a bachelor's degree and 5 years' community working experience in psychiatric treatment and care. Their average age was 36 years $($ s.d.=3.5). They attended three workshops over 12 days for $72 \mathrm{~h}$ run by qualified CBT trainers from Hong Kong (R.M.K.N.), the UK (D.K. and D.T.) and the USA (Jesse H. Wright) plus one formal training session focusing on the brief CBT manual prior to the study.

Before the trial started, they were required to submit three case reports to the primary investigator (Z.-J.L.), who is a consultant psychiatrist with special expertise in CBT for psychosis. They were also asked to audiotape each session with the participants' permission. Peer supervision on the delivery of the brief CBT programme was held once a week in each centre during the study period. Therapists presented the case formulation, treatment plar, therapy process and questions for every CBT case to the experienced therapist (Z.-H.G.) once every 2 weeks, either on site or by videc
conference. In addition, the primary investigator (Z.-).L.) provider face-to-face supervision to all therapists once a month.

\section{Measures}

All participants were assessed at baseline, post-treatment and at 6- and 12-month follow-up by independent raters masked to treatment allocation. All yaters were experienced researchers and received intensive interrater reliability training by watching videos before the study conmenced They also met every 3 months to receive this training again and discussed rating problems throughout the study.
The primary outcome measures included the PANSS ${ }^{15}$ and the
Schedule for Assessing Insight (SAI). ${ }^{8}$ The Chinese version of the Schedule for Assessing Insight (SAI) ${ }^{8}$ The Chinese version of the
PANSS is a 30 -item, semi-structured rating scale administered by clinicians used to assess the presence and severity of schizophrenic symptoms. 9 All items are scored between 1 (absent) and 7 (very severe) on a seven-point Likert scale and divided into three dimensions: positive, negative and general psychopathology symptoms. This scale has been shown to have good validity and reliability. ${ }^{15,19}$ A $25 \%$ or greater improvement in the total PANSS score between baseline and end-point was defined as a clinically significant response. ${ }^{11}$ Relapse was defined as admission to hospital, attempted suicide or deterioration, with one or more of the four psychotic symptoms on the PANSS (items P2, P3, P5 and G9) rated as 6 (severe) or 7 (very severe) or two or more of the psychotic symptoms rated as 5 (moderately severe). ${ }^{10}$ The $\mathrm{SAI}^{18}$ was used to evaluate insight in three dimensions: awareness of their illness, ability to relabel psychotic experiences correctly and attitudes to treatment. Each dimension consists of two or three questions that are scored on a three-point Likert scale from
0 (no insight) to 2 (good insight), with the total score ranging from 0 to 14 . Here, we used the Chinese version, which has good psychometric properties. ${ }^{20}$

Our secondary outcome measures included the Personal and Social Performance scale (PSP) $)^{21,22}$ and the Beck Depression Inventory-II (BDI-II). ${ }^{23}$ Social functioning was assessed using the Chinese version of the PSP, ${ }^{21,22}$ a 100-point, single-item rating scale based on an interview that assesses patients' personal and social functioning in four areas: socially useful activities, personal and social relationships, self-care, as well as disturbing and aggressive behaviours. Higher scores on the PSP total are indicative of better functioning. The scale showed good internal consistency (Cronbach's $\alpha=0.84$ ) and construct validity, with statistically significant correlations with the Global Assessment of Functioning scale. ${ }^{22}$ The BDI-II is 21-item self-report questionnaire measuring the intensity of depressive symptoms. ${ }^{23}$ Each item ranges from 0 to 3 points and indicates the severity of the depressive symptoms. The higher the score displayed, the greater the intensity of symptoms. It is positively correlated with the Hamilton Rating Scale for Depression with a Pearson $r$ of 0.71 . $^{23}$ It has also been demonstrated to have good reliability and validity for Chinese population samp

\section{Statisticar analysis}

Independent-samples t-test, Mann-Whitney $U$-test and Pearson's $x^{2}$-test were conducted to describe the demographic characteristics of each sample. The measury outcomes were analysed in two ways: intention-to-treat (ITT) and per-protocol. Mixed-model analysis was used for the ITT approach and the models were fixed main effects for treatment, time and baseline, and random effects for participants. Repeated-measures analysis of variance (ANOVA) was employed to compare the two groups over time on the yariables, with baseline scores as covariates. The mean doses of standarelised chlorpromazine equivalent at any point in both groups were compared using the independent samples $t$-test. In addition, effect sizes (Cohen's $d$ ) for all variables were calculated as indicators of the observed changes within groups. ${ }^{25}$ Two-tailed tests were used in all analyses with the significance level set at 0.05 . All analyses were performed using the Statistical Package for the Social Sciences (SPSS) Version 20.0.

\section{Results}

\section{Recruitment and sample characteristics}

A total of 220 patients were recruited for the study and randomly assigned to one of the two treatment arms. Of the patients, 89 (40.5\%) had paranoid-type, $105(47.7 \%)$ had undifferentiatedtype, $24(10.9 \%)$ had residual-type and $2(0.9 \%)$ had simple-type schizophrenia. As shown in Table 1, the two groups were well matched in all baseline demographic characteristics.

As seen in Fig. 1, 26 (9.4\%) of 276 referrals declined participation. Patients assigned to brief CBT received a mean of 6.5 sessions (range 2-8; s.d.=1.7). In total, $86(78.2 \%)$ of the 110 patients had at least six sessions, suggesting good adherence to CBT. In the brief CBT group, 94 (85.5\%) of the 110 participants completed the 64-week trial, whereas in the TAU group, 96 $(87.3 \%)$ of the 110 participants completed the whole trial. There was no significant difference between the two groups in terms of the proportion and demographic characteristics of participants failing to complete the assessment at any time point.

\section{Assessment of treatment efficacy}

In this study, the interrater reliabilities (intraclass correlation coefficients) of all the scales were above 0.8. The means and 


\begin{tabular}{|c|c|c|c|c|c|c|}
\hline \multirow[b]{2}{*}{ Variables } & \multirow{2}{*}{$\begin{array}{l}\text { Brief CBT group } \\
\quad(n=110)\end{array}$} & \multirow{2}{*}{$\begin{array}{l}\text { TAU group } \\
(n=110)\end{array}$} & \multicolumn{4}{|c|}{ Statistics } \\
\hline & & & $t$ & $z$ & $\chi^{2}$ & $P$ \\
\hline Age, years: mean (s.d.) & $34.94(10.50)$ & $37.16(9.89)$ & -1.620 & & & 0.107 \\
\hline Education, years: mean (s.d.) & $12.88(2.62)$ & $12.29(2.14)$ & 1.831 & & & 0.068 \\
\hline Duration of illness, months: mean (s.d.) & $137.27(101.27)$ & $163.29(116.76)$ & -1.766 & & & 0.079 \\
\hline Previous admissions, median (range) & $2(0-12)$ & $2(0-11)$ & & -0.762 & & 0.446 \\
\hline Gender, male: $n$ (\%) & $50(45.5)$ & $53(48.2)$ & & & 0.164 & 0.685 \\
\hline Ethnic group, Han: $n(\%)$ & $102(92.7)$ & $104(94.5)$ & & & 0.305 & 0.581 \\
\hline Living status, independent: $n$ (\%) & $10(9.1)$ & $16(14.6)$ & & & 1.578 & 0.210 \\
\hline Marital status, single: $n(\%)$ & $89(80.9)$ & $78(70.9)$ & & & 3.008 & 0.083 \\
\hline Unemployment, $n$ (\%) & $84(76.4)$ & $81(73.6)$ & & & 0.218 & \\
\hline
\end{tabular}

standard deviations of all the outcome measure scores at each time point are given in Table 2. The two groups were well matched in terms of their baseline symptom profiles, with the exception of the severity of depression. The BDI-II score in the brief CBT group was significantly higher than that in the TAU group $(t(218)=2.057, P=0.041)$ at baseline. Table 2 also shows the main effects of time, group and time $\times$ group interaction on each outcome. The primary analysis was performed using the per-protocol approach. The ITT analysis showed the same pattern of results.

The main effect of time was found in the PANSS total and PANSS general subscales, suggesting that there was a significant decrease over the study period across conditions. There was a main effect of group, but there was no significant time $\times$ group interaction, indicating that the brief CBT group had significantly lower scores than the TAU group on overall symptoms (at 12 weeks, $F(1,178)=6.142, P=0.014$; at 64 weeks, $F(1,178)=10.938$, $P=0.001)$ and general psychopathology (at 12 weeks, $F(1,178)=$ $6.811, P=0.01$; at 38 weeks, $F(1,178)=4.249$,
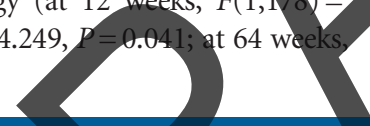

$F(1,178)=12.936, P<0.001$ ) throughout the trial (Fig. DS1 and
Fig. DS2).

There was a significant main effect of time for the PANSS positive subscale, but there was no significant time $\times$ group interaction or main effect of group, indicating that the two conditions resulted in signifieant reductions in positive symptoms, but with no significant difference across conditions. On the PANSS negative subscale a significant main effect of time was found, suggesting a decrease in both groups from baseline to 64 weeks. There was a marginally significant time $\times$ group interaction, which indicative of a strong trend of greater change in negative symptoms in the brief CBT group (at 64 weeks, $F(1,178)=$ $862, P=0.05$

Analyses of the SAI total score revealed a main effect of time, suggesting an increase over time in both conditions. The significant time $\times$ group interaction showed that the brief CBT group had significantly better insight than the TAU group (at 12 weeks, $F(1,178)=9.120, \quad P=0.003$; at 64 weeks, $F(1,178)=$ $14.769, P<0.001)$ throughout the trial (Fig. DS3). The main effect

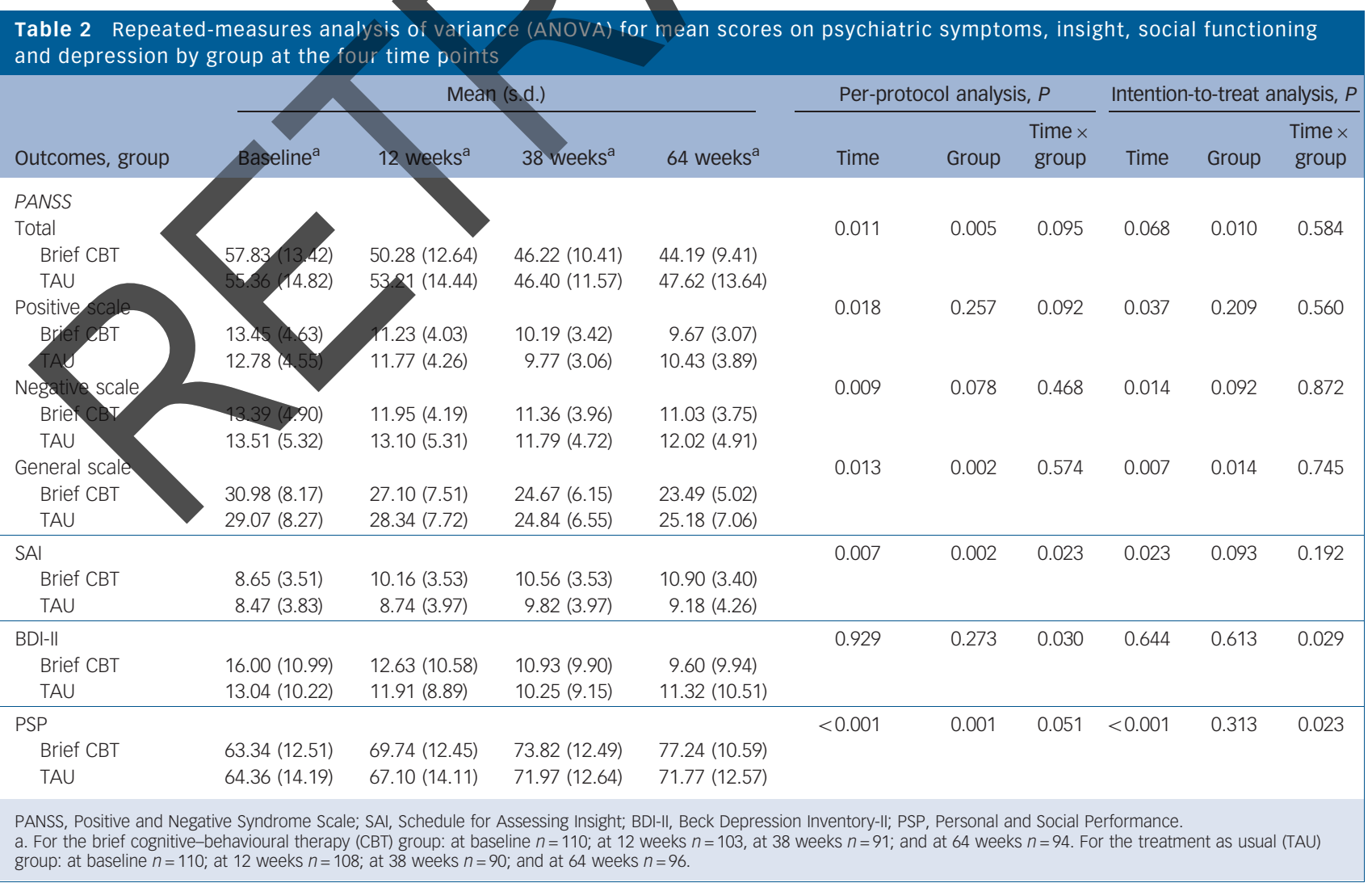


of time for the PSP score was significant, indicating that there was a significant increase in the PSP score across conditions. However, the main effect of group revealed that the brief CBT group had a significantly better level of functioning compared with the TAU group (at 12 weeks, $F(1,178)=4.322, P=0.039$; at 38 weeks, $F(1,178)=5.372, \quad P=0.022 ; \quad$ at 64 weeks, $\quad F(1,178)=15.004$, $P<0.001$ ) over the study period (Fig. DS4). With the baseline BDI-II score as a covariate, the time $\times$ group interaction was significant for the BDI-II, which demonstrated that brief CBT was beneficial for depressive symptoms at 64 weeks $(F(1,178)=4.721, P=0.031$, Fig. DS5 $)$.

Table 3 shows the effect sizes (Cohen's $d$ ) of change in the two groups at different time points. The brief CBT group demonstrated significantly larger effect sizes than the TAU group on the PANSS total, PANSS general, SAI, PSP and BDI-II scales.

\section{Clinical significance of symptom changes}

In accordance with the principle of ITT and with the criterion of a $25 \%$ or greater reduction on the PANSS total score, $37.3 \%$ (41/110) of those in the brief CBT group experienced a clinically significant improvement compared with only $19.1 \%$ (21/110) of those in TAU alone group, and this difference was statistically significant $\left(\chi^{2}=8.983, P=0.003\right)$. The number needed to treat $(\mathrm{NNT})^{26}$ for improvement in overall symptoms was $6(95 \% \mathrm{CI}$ 4-13), which means that for every six patients treated with brief CBT there was one extra good clinical response over TAU.

By the end of the trial, eight participants $(7.27 \%, 8 / 110)$ in the brief CBT group and ten participants $(9.09 \%, 10 / 110)$ in the TAU group had relapsed, two participants $(1.82 \%, 2 / 110)$ in the brief CBT group and three participants $(2.73 \%, 3 / 110)$ in the TAU group had been admitted to hospital; all the above differences between the two groups were not statistically significant $\left(\chi^{2}=0.242, P=0.623\right.$; Fisher's value 1.000)

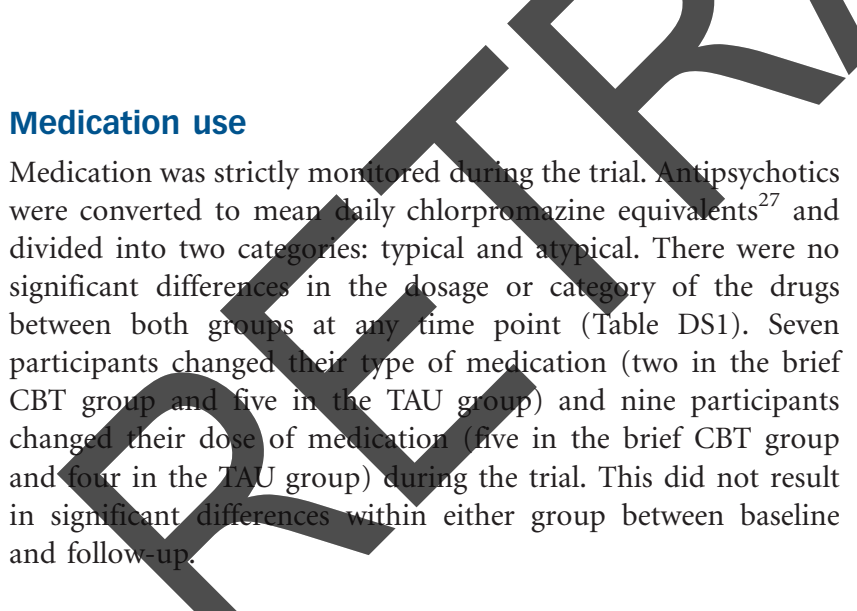

\section{Discussion}

\section{Main findings}

This is the first multicentre, large sample, RCT to have been undertaken using a brief CBT manual for the treatment of schizophrenia in the Chinese community. The results of this trial show that brief CBT has positive effects on patients in the community with schizophrenia. Brief CBT had many benefits on overall symptoms and general symptoms after acute treatment and the effects persisted in the 1-year follow-up period. Considering no superiority of adjunctive brief CBT on positive and negative symptoms in this study, the advantage in overall symptoms may be mainly from improvements in general psychopathology. This result is consistent with the findings of Morrison et al, who found that CBT could significantly alleviate overall symptoms and general symptoms in patients with schizophyenia who refused to take antipsychotic drugs. ${ }^{28}$ The short-term positive effects may be because of both non-specific factors (such as regular contact with clinicians) and the specific methods of CBT such as psychoeducation and normalisation, development of rational explanations, behavioural experiments and relaxation training. However, the superior effects of brief $\mathrm{CB}$ T over TAU in the long term may be because the CBT skills learned in the sessions can be practised repeatedly by the patients and have a sustained effect, as was shown in the 1-year follow-up period.

Past studies observed better outcomes in positive symptoms in those treated with CBT, ${ }^{29,30}$ but our study found no superiority of brief CBT for positive symptoms. The manual developed by us mainly focused on distress and safety behaviours that may be naintenance factors in hallucinations and delusions. ${ }^{31}$ An approach that challenges delusional beliefs or voices more directly might be a beneficial addition to the present programme. In addition, it seems that more time and expert therapists delivering CBT would be required to improve positive symptoms significantly. A similar study comparing the efficacy of brief CBT plus TAU with that of TAU using the Negative Symptom Rating Scale $(\mathrm{NSRS})^{32}$ found that brief CBT had modest effects on negative symptoms at 1-year follow-up, ${ }^{12}$ but our study found only a strong trend for greater change in those receiving brief CBT. The reason for this slight difference may be that CBT only has a small effect size on negative symptoms ${ }^{6}$ and the measurement instrument (PANSS) used here may not be as sensitive as the NSRS.

Our study also highlights the role of brief CBT in improving patients' insight. This result is consistent with Turkington et al, who reported a statistically significant improvement in overall insight after a brief CBT intervention delivered by trained nurses to patients with schizophrenia in the community. ${ }^{11}$ CBT techniques such as psychoeducation, normalisation and behavioural experiments help to improve patient awareness of disease and

\begin{tabular}{|c|c|c|c|c|c|c|}
\hline & \multicolumn{2}{|c|}{ Pre-12 weeks, group } & \multicolumn{2}{|c|}{ Pre-38 weeks, group } & \multicolumn{2}{|c|}{ Pre-64 weeks, group } \\
\hline & Brief CBT & TAU & Brief CBT & TAU & Brief CBT & TAU \\
\hline \multicolumn{7}{|l|}{ Positive and Negative Syndrome Scale } \\
\hline Total & $0.71 *$ & 0.26 & 0.91 & 0.76 & $1.21 * * *$ & 0.65 \\
\hline Positive scale & 0.60 & 0.35 & 0.67 & 0.69 & 0.81 & 0.63 \\
\hline Negative scale & 0.43 & 0.13 & 0.46 & 0.30 & 0.61 & 0.30 \\
\hline General scale & $0.66^{*}$ & 0.09 & $0.91 *$ & 0.51 & $1.14^{* * *}$ & 0.47 \\
\hline Schedule for Assessing Insight & $0.57^{* *}$ & 0.10 & 0.52 & 0.34 & $0.65^{* * *}$ & 0.13 \\
\hline Personal and Social Performance Scale & $0.76^{*}$ & 0.21 & $0.89 *$ & 0.65 & $1.01 * * *$ & 0.51 \\
\hline Beck Depression Inventory-II & 0.36 & 0.17 & 0.49 & 0.31 & $0.65^{\star}$ & 0.16 \\
\hline
\end{tabular}


symptom identification ability, ${ }^{33}$ alleviate their disease shame ${ }^{34}$ and increase their overall level of self-knowledge. ${ }^{35}$

It is generally believed that participants who demonstrated improved insight into having a mental illness tend to become depressed. ${ }^{36}$ However, in our study, the depressive symptoms of patients who received brief CBT did not get worse but significantly improved instead. So this trial demonstrated the effectiveness of brief CBT for the emotion management of patients, which is similar to both Turkington et al's and Waller et al's findings that brief or low-intensity CBT led to a great improvement in depression and distress. ${ }^{11,13}$

Our study has also demonstrated an effect of brief CBT on the social functioning of people with schizophrenia, which compares well with previously published studies. For instance, Granholm et $a l^{37}$ found that older people with chronic schizophrenia were able to learn new skills and showed improved functioning 1 year after the CBT ended. Grant $e t a l^{38}$ reported that patients treated with CBT showed more improvement in global functioning from baseline to 18 months compared with standard treatment. The beneficial change may be as a result of a decline of psychiatric symptoms and depression, but specific techniques such as behaviour activation and problem-solving also benefit patients' social functioning. ${ }^{28,39}$

In our study, both groups of patients received case management and their medication adherence was good, so the 1-year recurrence and hospital admission rates were both low. During the study period, there were no significant differences in medications or dosages across conditions, so the greater improvement in symptoms, insight and social functioning in the brief CBT group cannot be attributed to medication. However, compared with TAU, additional CBT did not reduce the recurrence rate further, perhaps because of the lack of maintenance treatment in the brief programme administered in this study. ${ }^{40}$

\section{Strengths and limitations}

The methodological strengths of the sample size, the randomised allo of skills training using a m supervision, the masked assessment of outcomes, the monitoring of medication throughoty the study and the per-protocol combined with ITT analysis. However, this study also has some limitations. For instance, patients were recruited by repeatedly canvassing local services for referrals rather than by systematically screening patient populations. ${ }^{29}$ The inclusion criteria were relatively stringent and it is possible that some patients with more difficult presentations may have been excluded. We did not use the Cognitive Therapy Scale ${ }^{41}$ to check treatment fidelity, because such a scale has not been developed in China. Further, as there was no active control group, non-specific factors cannot be ruled out as the reason for the effect of brief CBT. However, the long-term effect of brief CBT is unlikely to be because of those non-specific factors as they only have a temporary effect. ${ }^{9}$

\section{Implications}

Brief CBT not only reduced patients' overall symptoms and general psychopathology but also improved their insight, mood and social functioning. This study suggests that community psychiatrists and nurses who receive a short-term training programme and regular supervision can effectively deliver brief CBT to patients with schizophrenia in primary healthcare. Brief CBT may be a feasible intervention for patients in the community in China.

\section{Funding}

This work was funded by the Capital Medical Development Programme (Grant Number: 20091050), the China-WHO 2010-2011 Collaborative Programme (Grant Number: WPCHN1003566) and the Beijing Hospital Authority Programme (Grant Number: XMLX201403), which were not involved in the study design, data collection, analysis or interpretation.

\section{Acknowledgements}

We thank all the patients who participated in this research. We are very grateful to all the therapists in the study. We would also like to acknowledge Jesse H. Wright (University of Louisville, USA) for his contribution to the training in CBT for psychosis.

Zhi-Hua Guo, MD, Zhan-Jiang Li, MD, PhD, Yun Ma, MSC, Department of Clinica Psychology \& Beijing Key Lab of Mental Disorders, Belling Anding Hospital, Capital Medical University, and Centre of Schizophrenia, Beijing Institute for Brain Disorders, Beijing, People's Republic of China; Jing Sun, PhD,

Queensland and School of Medicine, G

Jun-Hua Guo, MD, Department of $P$

Jun-Hua Guo, MD, Department of P
Medical University, Beijing, People's

Health Prevention Hospital of Haid

Zhi-Qiang Wang, MD, Dongchen

Centre, Beijing, People's Republic

of China; Hui-Li Xu, MD, Chaoyang District

Centre for Mental Disease Control ard Prevention, Beijing, People's Republic of China:

Roger M. K. Ng, MD, PhQ. Department of Psychiatry, Koylogh Hospital, Hong Kong,

People's Republic of China; Douglas Turkington, MD, MRCPsych, Newcastle

University, Newcastle-on-Tyne, UK; David Kingdon, MD, MRCPSych, Department
of Psychiatry, Uni ersity of Southampton, Southampton, UK

Correspondence: Zkan-Jiang Li, MD, PhD, Professor of Psychiatry \& Clinical

Psychology, Departmen of Clinical Psychology, Beijing Anding Hospital, Capita

Medical University, No. 5 Ankang Hutong, Deshengmen Wai, Xicheng District,

Beiiing 100088, People's Republic of China. Email: lizhj8@ccmu.edu.cn

First received 21 Feb 2016, finà revision 17 Sep 2016, accepted 19 Sep 2016

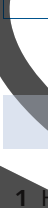

1 Henry LP, Amminger GP, Harris MG, Yuen HP, Harrigan SM, Prosser AL, et al. The EPPIC follow-up study of first-episode psychosis: Ionger-term clinical and functional outcome 7 years after index admission. J Clin Psychiatry 2010; 71: 716-28

2 Chen $\mathrm{CH}$, Shen YC, Zhang WX, Li SR, Huang YQ, Wang JR, et al. Epidemiological survey on schizophrenia in 7 areas of China [in Chinese] Chin J Psychiatry 1998; 31: 72-4.

3 Ferrari AJ, Saha S, McGrath JJ, Norman R, Baxter AJ, Vos T, et al. Health states for schizophrenia and bipolar disorder within the Global Burden of Disease 2010 Study. Popul Health Metr 2012; 10: 1.

4 Olivares JM, Sermon J, Hemels M, Schreiner A. Definitions and drivers of relapse in patients with schizophrenia: a systematic literature review. Ann Gen Psychiatry 2013; 12: 32.

5 Ascher-Svanum H, Faries DE, Zhu B, Ernst FR, Swartz MS, Swanson JW. Medication adherence and long-term functional outcomes in the treatment of schizophrenia in usual care. J Clin Psychiatry 2006; 67: 453-60.

6 Wykes T, Steel C, Everitt B, Tarrier N. Cognitive behavior therapy for schizophrenia: effect sizes, clinical models, and methodological rigor. Schizophr Bull 2008; 34: 523-37.

7 National Institute for Health and Care Excellence. Core Interventions in the Treatment and Management of Schizophrenia in Adults in Primary and Secondary Care. National Collaborating Centre for Mental Health, 2009.

8 Kreyenbuhl J, Buchanan RW, Dickerson FB, Dixon LB. The schizophrenia patient outcomes research team (PORT): updated treatment recommendations 2009. Schizophr Bull 2010; 36: 94-103.

9 Li ZJ, Guo ZH, Wang N, XU ZY, Qu Y, Wang XQ, et al. Cognitive-behavioural therapy for patients with schizophrenia: a multicentre randomized controlled trial in Beijing, China. Psychol Med 2015; 45: 1893-905.

10 Xiang Y-T, Weng Y-Z, Li W-Y, Gao L, Chen G-L, Xie L, et al. Efficacy of the Community Re-Entry Module for patients with schizophrenia in Beijing, China: outcome at 2-year follow-up. Br J Psychiatry 2007; 190: 49-56.

11 Turkington D, Kingdon D, Turner T. Effectiveness of a brief cognitivebehavioural therapy intervention in the treatment of schizophrenia. Br J Psychiatry 2002; 180: 523-7.

12 Turkington D, Kingdon D, Rathod S, Hammond K, Pelton J, Mehta R. Outcomes of an effectiveness trial of cognitive-behavioural intervention by mental health nurses in schizophrenia. Br J Psychiatry 2006; 189: 36-40. 
13 Waller H, Garety PA, Jolley S, Fornells-Ambrojo M, Kuipers E, Onwumere J, et al. Low intensity cognitive behavioural therapy for psychosis: a pilot study. J Behav Ther Exp Psychiatry 2013; 44: 98-104.

14 World Health Organization. The ICD-10 Classification of Mental and Behavioural Disorders: Clinical Descriptions and Diagnostic Guidelines. WHO, 1992.

15 Kay SR, Fiszbein A, Opler LA. The Positive and Negative Syndrome Scale (PANSS) for schizophrenia. Schizophr Bul 1987; 13: 261-76.

16 Guo ZH, Li ZJ, Ma Y, Zhou YL, Chao WM, Guo JH, et al. A pilot study on the effect of brief cognitive behavioural therapy combined with routine treatment for schizophrenia in communities of Beijing [in Chinese]. Chin J Psychiatry 2015; 48: 331-8.

17 Chen M, Wu G, Wang Z, Yan J, Zhou J, Ding Y, et al. Two-year prospective case-controlled study of a case management program for communitydwelling individuals with schizophrenia. Shanghai Arch Psychiatry 2014; 26: 119-28.

18 David AS. Insight and psychosis. Br J Psychiatry 1990; 156: 798-808.

19 Si TM, Yang JZ, Shu L, Wang XL, Kong QM, Zhou M, et al. The reliability, validity of PANSS and its implications [in Chinese]. Chin Ment Health 2004; 18: $45-7$.

20 Xu ZY, Guo ZH, Fu ZY, Wang N, Zhang Y. Reliability and validity of the Chinese version of the schedule for assessment of insight [in Chinese]. Chin J Behav Med Brain Sci 2013; 22: 752-4.

21 Morosini P, Magliano L, Brambilla L, Ugolini S, Pioli R. Development, reliability and acceptability of a new version of the DSM-IV Social and Occupational Functioning Assessment Scale (SOFAS) to assess routine social functioning. Acta Psychiatr Scand 2000; 101: 323-9.

22 Tianmei S, Liang S, Yun'ai S, Chenghua T, Jun $Y$, Jia $C$, et al. The Chinese version of the Personal and Social Performance Scale (PSP): validity and reliability. Psychiatry Res 2011; 185: 275-9.

23 Beck AT, Steer RA, Ball R, Ranieri W. Comparison of Beck Depression Inventories-IA and -II in psychiatric outpatients. J Pers Assess 1996; 67: 588-97.

24 Wang Z, Yuan CM, Huang J, Li ZZ, Chen Y, Zhang HY, et al. Reliability and validity of the Chinese version of Beck Depression Inventory-II among depression patients [in Chinese]. Chin Ment Health 2011; 25: 476-80

25 Cohen J. A power primer. Psychol Bull 1992; 112: 155-9.

26 Pinson L, Gray GE. Psychopharmacology: number needed to treat: underused measure of treatment effect. Psychiatr Serv 2003; 54: 14

27 Lehman AF, Lieberman JA, Dixon LB, McGlashan T et al. Practice guideline for the treatment of pa second edition. Am J Psychiatry 2004; 161

28 Morrison AP, Turkington D, Pyle M, Spen Cognitive therapy for people with schizophrenia sraban taking antipsychotic drugs: a single disorders not 2014; 383: 1395-403.

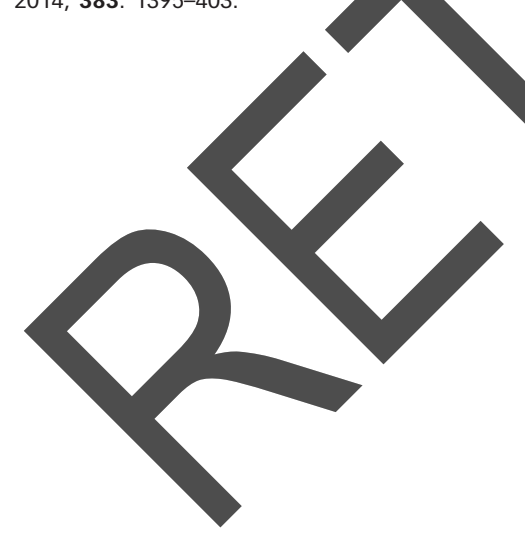

29 Tarrier N, Yusupoff L, Kinney C, McCarthy E, Gledhill A, Haddock G, et al. Randomised controlled trial of intensive cognitive behaviour therapy for patients with chronic schizophrenia. BMJ 1998; 317: 303-7.

30 Zimmermann G, Favrod J, Trieu V, Pomini V. The effect of cognitive behavioral treatment on the positive symptoms of schizophrenia spectrum disorders: a meta-analysis. Schizophr Res 2005; 77: 1-9.

31 Freeman D, Dunn G, Startup H, Pugh K, Cordwell J, Mander H, et al. Effects of cognitive behaviour therapy for worry on persecutory delusions in patients with psychosis (WIT): a parallel, single-blind, randomised controlled trial with a mediation analysis. Lancet Psychiatry 2015; 2: 305-13.

32 Hansen $L$, Turkington $D$, Kingdon $D$, Smith $P$. Brief rating instrument for assessment of negative symptoms: derived from the comprehensive Psychopathological Rating Scale (CPRS). Int J Psychiatry Clin Pract 2003; 7: 113-6.

33 Rathod S, Kingdon D, Smith $\mathrm{P}$, Turkington D. Insight into schizophrenia: the effects of cognitive behavioural therapy on the components of insight and association with sociodemographics-data on a previously publisked randomised controlled trial. Schizophr Res 2005; 74; 211-9.

34 Gerlinger G, Hauser M, De Hert M, Lacluyse K, Wampers M, Correll CU. Personal stigma in schizophrenia spectrum disorders: a systematic review of prevalence rates, correlates, impact and interventions. World Psychiatry 2013; 12: 155-64

35 Bedford NJ, David AS. Denial of illness in schizophrenia as a disturbance of self-reflection, selfperception and insight. Schizophr Res 2014; 152: 89-96.

36 Iqbal Z, Birchwood M, Chadwick $P$, Trower P. Cognitive approach to depression and suicidal thinking in psychosis 2 . Testing the validity of a social ranking model. Bry Psychiatry 2000; 177: 522-8.

37 Granholm E, McQuaid JR, Mcclure FS, Link PC, Perivoliotis D, Gottlieb JD, et al Randomized controlled trial of cognitive behavioral social skills training for older people with schizophrenia: 12-month follow-up. J Clin Psychiatry 2007; 68: 730-7.

8 Grant PM, Huh GA, Perivoliotis D, Stolar NM, Beck AT. Randomized trial to evaluate the efficacy of cognitive therapy for low-functioning patients with schizophrenia. Arch Gen Psychiatry 2012; 69: 121-7.

39 Led

9 Lecomte-T. PSychosis patients refusing antipsychotic medicine could benefit from CBT in terms of both symptom reduction and social functioning. Evid Based Ment Health 2015; 18: 18

40 Tarrier $\mathrm{N}$, Lewis $\mathrm{S}$, Haddock $\mathrm{G}$, Bentall $\mathrm{R}$, Drake $\mathrm{R}$, Kinderman $\mathrm{P}$, et al. Cognitive-behavioural therapy in first-episode and early schizophrenia: 18-month follow-up of a randomised controlled trial. Br J Psychiatry 2004; 184: $231-9$

41 Blackburn IM, James IA, Milne DL, Baker C, Standart S, Garland A, et al. The revised cognitive therapy scale (CTS-R): psychometric properties. Behav Cogn Psychother 2001; 29: 431-46. 